\title{
SNAPSHOTS OF HIGH-LATITUDE ELECTRODYNAMICS USING VIKING AND DMSP/F7 OBSERVATIONS
}

G.T. Marklund et al.

February 1988

Department of Plasma Physics The Royal Institute of Technology S-100 44 Stockholm, Sweden 


\section{SNAPSHOTS OF HIGH-LATITUDE ELECTRODYNAMICS USING VIKING AND DMSP/F7 OBSERVATIONS}

G.T. Marklund ${ }^{1}$, L.G. Blomberg ${ }^{1}$, K. Stasiewicz ${ }^{2}$, J.S. Murphree ${ }^{3}$, R. Pottelette ${ }^{4}$, L.J. Zanetti ${ }^{5}$, T.A. Potemra ${ }^{5}$, D.A. $\operatorname{Hardy}^{6}$ and F.J. Rich ${ }^{6}$

1) Department of Plasma Physics, The Royal Institute of Technology, Stockholm, Sweden

2) Swedish Institute of Space Physics, Kiruna, Sweden

3) Department of Physics, University of Calgary, Calgary, Alberta, Canada

4) Centre de Recherches en Physique de l'Environnement Terrestre et Planétaire, Saint-Maurdes-Fossés, France

5) Applied Physics Laboratory, The Johns Hopkins University, Laurel, Maryland, USA

6) Space Physics Division, Air Force Geophysics Laboratory, Bedford, Massachusetts, USA

Abstract. Simultaneous observations by the Viking and the DMSP/F7 satellites have been used in a new method to obtain snapshot pictures of the auroral electrodynamics. In particular, an "instantaneous" global equipotential (or convection) pattern is calculated using field-aligned current and conductivity distributions that are qualitatively consistent with the Viking auroral imager data and quantitatively consistent with magnetic field and particle data from the two satellites. This convection pattern which is of the normal two-cell type, with a weak dusk cell and a strong, elongated crescent-shaped dawn cell (consistent with positive IMF $B_{y}$ ) agrees well with the Viking electric field data. The model and the observed potential profiles agree nicely along the entire Viking orbit except for two intervals above acceleration regions where deviations are to be expected (due to parallel electric fields). These regions are characterized by: U-shaped potential minima, upward field-aligned currents, upgoing ion beams and relatively intense AKR. The model results are thus consistent with the Viking observations not only on a global scale but also on the scale size of the auroral acceleration regions. The corresponding convection in the magnetosphere is illustrated by a simple projection of the deduced two-cell convection pattern to the equatorial plane. From this the instantaneous location of the plasmapause is inferred. 


\section{Introduction}

In our efforts to better understand the magnetosphere-ionosphere system a major problem is to provide an acceptable coverage by simultaneous observations within the large volume to be studied. The high-latitude ionosphere acts in many respects as a projection screen for processes taking place in the outer magnetosphere and boundary regions. Much can therefore be learnt from studies of the electrodynamics of the high-latitude ionosphere, which is a relatively small and easily accesible region. Joint efforts to combine simultaneous observations by different spacecraft and/or ground-based facilities have become relatively common recently. The problem to be solved is how to utilize the information from the various observations to reconstruct, on a global scale, the instantaneous distributions of all relevant electrodynamical parameters in a selfconsistent manner.

Kamide et al. [1986] used images of auroral emissions observed with Dynamics Explorer 1 to calculate high-latitude ionospheric conductivities. By combining these with simultaneous ground-based magnetometer data they were able to estimate on a global scale the distributions of ionospheric electric fields and currents, field-aligned currents and Joule heating with a time resolution of $12 \mathrm{~min}$.

In this paper a new and different approach to this problem will be described using observations from two almost simultaneous auroral oval crossings by the Viking and DMSP/F7 satellites. A brief summary of this new technique, first presented by Marklund et al. [1987], (from here on referred to as Paper 1), will be given below:

1. The quiet-time diffuse background auroral oval is represented by the quiet-time field-aligned current distribution from Iijima and Potemra [1976a, 1978] and a corresponding quiet-time conductivity distribution described below.

2. UV-imaging of the auroral oval is used to obtain a reference frame for the other observations and in particular to locate the regions of active intense auroras. These are clearly associated with enhanced upward field-aligned currents and ionospheric conductivities. The input distributions are obtained by a superposition of the quiet-time and active distributions.

3. In situ satellite measurements are used both to calibrate the input current and 
conductivity distributions and to test the output potential distribution along the ionospheric footpoints of the satellites.

In Paper 1 only Viking data was used to calibrate the input and to check the results. However, even for this case, relatively accurate and realistic global distributions of electric fields, currents and conductivities were obtained (cf. Figure 4 in Paper 1). In the present paper, a number of improvements as compared to Paper 1 have been made, of which the most important ones are:

1) Inclusion of DMSP/F7 data of magnetic fields and particle fluxes which allows the model input data to be calibrated also along the noon-midnight orbit of the DMSP/F7 satellite.

2) More accurate estimates of the downward electron energy flux using appropriate scaling factors between Viking and the ionosphere. More accurate representation of the auroral distribution given by the UV-image, taking into account also the finer-scale structures.

3) A two-dimensional comparison between the model convection pattern and $\mathbf{E} \times \mathbf{B}$ - drifts calculated from the Viking electric field data.

4) A detailed investigation of the auroral acceleration regions including electric field, current, particle and wave signatures.

\section{Basic Equations}

The basic equation to be solved is the elliptical equation governing the horizontal distribution of the ionospheric electric potential, $\Phi$ :

$$
\nabla \cdot(\boldsymbol{\Sigma} \cdot \nabla \Phi)=j_{\|} \cdot \sin I
$$

where $j_{\|}$is the field-aligned current density (positive upwards), I the inclination of the Earth's magnetic field and $\boldsymbol{\Sigma}$ the horizontal conductivity tensor given by:

$$
\boldsymbol{\Sigma}=\left[\begin{array}{cc}
\Sigma_{P} / \sin ^{2} I & \Sigma_{H} / \sin I \\
-\Sigma_{H} / \sin I & \Sigma_{P}
\end{array}\right]
$$

in which $\Sigma_{P}$ and $\Sigma_{H}$ are the height-integrated Pedersen and Hall conductivities. The electromotive force $\mathbf{v}_{\mathbf{n}} \times \mathbf{B}$ has been neglected since this contribution is small relative to that of $\mathbf{E}(=-\nabla \Phi)$, as recently demonstrated by Dynamics Explorer 
results [Killeen, private communication]. The calculation is performed in a spherical coordinate system and the magnetic field is represented by a dipole. The conductivity distribution is represented by the following model:

$$
\begin{gathered}
\Sigma_{P}=\sqrt{\Sigma_{0 P}^{2}+\Sigma_{g P}^{2}+\Sigma_{U V P}^{2}+\Sigma_{j_{\|} P}^{2}} \\
\Sigma_{H}=\sqrt{\Sigma_{0 H}^{2}+\Sigma_{g H}^{2}+\Sigma_{U V H}^{2}+\Sigma_{j_{\|} H}^{2}}
\end{gathered}
$$

(The quadratic form of Eqs.(3), (4) is a result of the linear relationship between conductivity and plasma density and quadratic relationship between density and recombination rate.) $\Sigma_{0 P(H)}$ represents the contribution from cosmic radiation and galactic EUV, and has a small constant value, $\Sigma_{g P(H)}$ a conductivity contribution having a gaussian latitudinal distribution representing the average diffuse auroral precipitation, $\Sigma_{U V P(H)}$ the contribution by solar EUV-radiation and $\Sigma_{j_{\|} P(H)}$ the enhanced conductivity associated with upward field-aligned currents caused by discrete auroral precipitation. The latter has been chosen to have the following form

$$
\Sigma_{j_{\|}}= \begin{cases}k(M L T) \cdot\left|j_{\|}\right|, & \text {for } j_{\|} \text {upward } \\ 0, & \text { for } j_{\|} \text {downward }\end{cases}
$$

where $k_{P(H)}$ is an MLT-dependent factor of proportionality having a lower value on the dayside than on the nightside to account for a softer dayside particle spectrum. A relationship between $\boldsymbol{\Sigma}$ and $j_{\|}$was also used by Mishin et al. [1986]. A more detailed description of the particular choice of conductivity model made and of the numerical solution is given elsewhere [Blomberg and Marklund, 1988a,b]. The inclusion of the conductivity term expressed by Eq.(5) above is made to obtain a selfconsistent relation between the distributions of field-aligned currents and conductivities. The values of the different conductivity terms have been chosen so as to give good large-scale agreement with empirical models of the auroral conductivity [e.g., Wallis and Budzinski, 1981] as well as good agreement along the two satellite trajectories with the conductivity calculated from measured data. As a boundary condition for the electric field we have assumed the potential to be zero at the magnetic equator. 


\section{Geophysical Situation During Viking Orbit 360}

Figure 1 shows the northern hemisphere auroral oval as viewed by the UVimager experiment on Viking at 07.24 UT during orbit 360, April 28, 1986. The auroral oval was crossed by Viking in roughly a dusk-dawn orbit and by DMSP/F7 in roughly a noon-midnight orbit as shown by the inserted ionospheric footpoints of the two satellites.

Three localized bright auroral structures can be recognized in the evening and midnight sector, the largest centered at about $65^{\circ}$ invariant latitude, 01.00 magnetic local time (MLT) with an east-west extent of roughly $20^{\circ}$. The western edge of the bright spot around 21.30 MLT was crossed by the DMSP/F7 satellite as will be shown below. Relatively bright dayside auroras are seen in the pre-noon sector whereas the remaining part of the auroral oval has a relatively smooth and regular luminosity distribution characteristic of the diffuse aurora.

For the dayside oval the auroral distribution remains relatively stable during this time, as indicated by the excellent agreement between the cusp currents measured by both Viking and DMSP/F7 about one hour apart (see below). Of the nightside auroral activity, the bright spot centered around 21.30 MLT was traversed by the DMSP/F7 satellite around 07.50 UT which allowed a calibration of the model input data for this part of the nightside oval within less than half an hour after the UV-image was taken. The 3 -hour $K_{P}$-index for this event ranged between $4^{-}$and 3. Unfortunately no IMP-8 interplanetary magnetic field data exist for this time period, but data from the Greenland magnetometer chain indicate that the IMF $B_{y}$ - component was positive during this event [E. Friis-Christensen, private communication].

\section{Results And Comparisons With Viking And DMSP/F7-Satellite Data}

Figure 2 shows the input $\Sigma_{P}$ and $j_{\|}$- distributions which are qualitatively consistent with the auroral image seen in Figure 1 and, as will be shown below, in excellent agreement with the Viking and DMSP/F7 data.

The Region 1 and Region 2 current patterns of Iijima and Potemra [1976a, 1978] representative for the diffuse quiet-time auroral oval, are seen to be modified by: 1) 
current enhancements within the bright auroral structures identified from Figure 1,2 ) cusp current sheets, inferred from the Viking magnetic field observations (see below) with location and extent in accordance with the observations by Iijima and Potemra [1976b]. The $\Sigma_{P}$ - distribution is seen to peak in the regions of upward field-aligned currents (cf. Eq.5) with intense maxima in the bright auroral forms. The ionization contribution from solar EUV gives rise to the daynight conductivity gradient.

The two projected orbits are seen to cross the pre-noon auroral oval (Region 1 and Region 2) relatively close and cross each other almost at the centre of the prenoon cusp current sheet. The one hour time separation between the two satellites at this location allows us to check the time stability of this pre-noon triple current sheet (see below). Note that the DMSP/F7 satellite passed through the intense bright auroral form around 21.30 MLT.

Figure 3 shows at the top, the model and calculated height-integrated Pedersen conductivity profiles, and at the bottom, the model and calculated field-aligned current profiles along the ionospheric projection of the Viking orbit. The contribution to $\Sigma_{P}$ from the measured particles has been estimated using that it is roughly proportional to the squareroot of the downward electron energy flux [Harel et al., 1981]. The field-aligned currents are estimated from the Viking magnetic field data presented in Figure 4 (bottom) together with the electric field (top). For a definition of the coordinate system used see Block et al. [1987]. The current values have been scaled so that they refer to the ionospheric level. Note the clear large-scale correlation between the orthogonal electric field and magnetic field components in Figure 4. This allows an independent estimate of the $\Sigma_{P}$ variation (denoted by circles in Figure 3) as demonstrated by Sugiura [1984].

As can be seen the $\Sigma_{P}$ - and $j_{\|}$- profiles calculated from the Viking data are very well represented by the model data both in terms of the relative magnitudes, widths and locations of the major structures. Note that the calculated conductivity always has local maxima in the regions of upward field-aligned currents. This field-aligned current-conductivity coupling is a characteristic feature in auroral electrodynamics and has been taken into account here by using the linear relationship between $j_{\|}$ 
and $\Sigma$, described by Eq.5.

Figure 5 shows in a similar fashion as in Figure 3 comparisons between the model conductivity and field-aligned current profiles and the corresponding estimates based on the DMSP/F7 data. Also here, the agreement is excellent concerning the large-scale variations. Consider for example the triple current sheet encountered around $07.40 \mathrm{UT}$. The location and the integrated values of the upand downflowing currents are very similar although the width of the model current is slightly greater. As mentioned above this triple current sheet was also traversed by the Viking satellite about one hour later. The magnitudes and signatures of the currents remain essentially the same during this one hour time period, with a value of $0.8-1 \mu \mathrm{A} / \mathrm{m}^{2}$ for the Region 2 upward current, $3 \mu \mathrm{A} / \mathrm{m}^{2}$ for the Region 1 downward current and about $2 \mu \mathrm{A} / \mathrm{m}^{2}$ for the upward cusp current. Thus for the region covered by the two satellites the geophysical conditions seem to have been relatively stable for the time period of interest here.

For the nightside auroral crossing the model conductivity and field-aligned current represents very well the auroral conditions prevailing at the time of the UVimage. The narrow structure at the poleward edge of the oval (07.50 UT) is, however, not represented in the model. This may be either just a local arc structure or it may represent the poleward part of a multiple current sheet associated with the Harang discontinuity. Adopting the former interpretation there is no reason to include small-scale structures in the model only along the satellite orbits where these can be detected and not elsewhere. The net current from these opposite currents is close to zero and will therefore not affect the results except very locally.

To summarize, the model input data are believed to rather accurately represent the real field-aligned current and conductivity distributions, as verified by the excellent agreement with the measurements along the two different satellite orbits.

The equipotential distribution, calculated using the numerical model described in Section 2 and the input distributions presented above, is presented in Figure 6 in an inertial frame of reference. Superposed on this we have for comparison plotted the $\mathbf{E} \times \mathbf{B}$ - drift vectors calculated from the Viking electric field (Figure 
4) assuming that $\mathbf{E} \cdot \mathbf{B}=0$. Note that the drift vectors are almost parallel to the convection streamlines everywhere except for two narrow regions as will be discussed below.

The calculated convection pattern is of the normal two-cell type with a weak dusk cell displaced toward noon and a more intense, longitudinally extended and crescent-shaped dawn cell characteristic for the prevailing positive IMF $B_{y}$. In between the convection streamlines form a throat-like pattern. Note the westward intrusion or clockwise rotation of the convection pattern close to midnight. Such a rotation is caused by the combined effect of intense field-aligned currents and polarization electric fields associated with the bright auroral intensifications there [Marklund et al., 1985] as well as the day-night conductivity gradient [Atkinson and Hutchison, 1978]. The polar cap potential drop is about $60 \mathrm{kV}$ in good agreement with empirical values for the prevailing $K_{P}$ - index [e.g., Foster et al., 1986] and directed roughly between 02 - 03 MLT and 15 MLT .

Figure 7 (bottom) shows the model and calculated potential profile along the ionospheric projection of the Viking orbit. This serves to demonstrate that the model results are not only qualitatively but also quantitatively in good agreement with the observations. Deviations occur, however, in two time intervals, namely around 07.20 UT and 08.30 UT where the high-altitude potential (Viking) have minima which are smaller or absent in the ionospheric (model) potential. It is evident from the measured field-aligned current profile (top), that intense upward currents are present in these regions. Furthermore these regions show evidence of upward acceleration of ions as indicated by the inserted shaded areas. This is examplified in Figure 8 which shows pronounced upward ion beams in the two highest energy channels $(0.52 \mathrm{keV}$ and $1.25 \mathrm{keV})$ between 07.20 and 07.23 UT. In the downward current region between $07.18 \mathrm{UT}$ and $07.20 \mathrm{UT}$, weak ion conics can be seen at the low energy channel of $60 \mathrm{eV}$. The electron data (not shown here) show only weak acceleration signatures and are thus consistent with the upward ion beams observed essentially above the acceleration region. Another piece of evidence that Viking passed above an auroral acceleration region centered around $07.20 \mathrm{UT}$ is given by the high frequency wave spectrogram in Figure 9 . 
The electric component is shown here for the time interval between 07.17.30 UT and 07.22.10 UT. Intense AKR-emissions centered around 07.19.40 UT with low frequency cutoffs between 350 and $400 \mathrm{kHz}$ are seen at the top well above the electron gyrofrequency indicated by the solid line at around $100 \mathrm{kHz}$. Note also the impulsive character of the low frequency hiss emissions around 07.19.55 UT, which is characteristic for the crossing of an arc structure. To summarize, the model output potential distribution agrees both qualitatively and quantitatively with the Viking observations, except in two regions where expected deviations occur, qualitatively consistent with upward parallel electric fields.

A simplified picture of the magnetospheric convection is illustrated in Figure 10 (right), which is a projection of the deduced ionospheric two-cell convection pattern (left) to the equatorial plane using a dipole magnetic field. The location of the last closed equipotential contour (heavy line) is seen to correspond relatively well with that of the average plasmapause boundary (dotted line) as given by Carpenter [1966]. (As the shape of the plasmapause varies a detailed correspondence is not to be expected.)

\section{Discussion}

UV-imaging of the aurora from polar orbiting satellites such as Dynamics Explorer I and Viking, represents an important new input to the study of auroral electrodynamics on a global scale. The images of the auroral oval provide not only a natural reference frame for the other observations, but also valuable information on various electrodynamical parameters. Active regions such as bright auroral structures are clearly associated with enhanced conductivities and fieldaligned currents. To obtain representative estimates of these parameters for the diffuse auroral oval (background) it was found better to use a realistic conductivity model and the measured average field-aligned current distributions of Iijima and Potemra [1976a, 1978] representative for quiet time conditions, than to rely on an uncertain relationship between conductivities and UV intensity.

An important and new feature of the model presented here is that the fieldaligned current and conductivity patterns are consistent with each other in the sense that the conductivity peaks in regions of upward field-aligned currents as- 
sociated with the discrete aurora. The exact form of such a correspondence is a very complicated problem since the precipitating particles creating enhanced ionization are typically more energetic than those carrying the major part of the upward field-aligned currents. Qualitatively such a correspondence is, however, well confirmed from observations over auroral arcs. Lyons et al., [1979] found that when a field-aligned acceleration voltage (V) could be inferred from the electron spectra, the net downward electron energy flux generally varied as $\mathrm{V}^{2}$ while the net upward field-aligned current was generally proportional to V. Since $\Sigma_{P}$ is roughly proportional to the square-root of the electron energy flux (see Section 2 above) the relations above imply a direct proportionality between $\Sigma_{P}$ and $j_{\|}$. A correspondence between these two parameters is also evident from Figures 3 and 5. which justifies our choice of the linear relationship described by Eq.(5).

The new method described in this paper is still under development and refinements of some of the details in the model are to be expected as soon as we have gained more experience from several different events. The criteria used in the selection of a suitable Viking orbit for a first study was that the entire auroral oval should be visible by the imager and that the substorm activity should keep a constant and fairly moderate level during the satellite transit across the oval. Orbit 360 during which the $K_{P}$ - index varied between $4^{-}$and 3 was found to meet these various requirements reasonably well. Note that the results are to be representative for the auroral situation illustrated by the UV-image in Figure 1. To accomplish this we require that the auroral electrodynamical conditions as probed by the two satellites along their different orbits should remain similar to that of the UV-image during the respective satellite transit times over the oval. For the dayside auroral activity as probed by the Viking satellite this requirement is believed to be fulfilled, supported by the almost identical triple field-aligned current sheets observed by the DMSP/F7 satellite at 07.40 UT (cf. Figure 5) and by the Viking satellite about one hour later (cf. Figure 3). For the nightside auroral oval the requirement is that the activity should remain roughly unchanged until the DMSP/F7 satellite at $07.50 \mathrm{UT}$ crossed the eveningside auroral oval. We have assumed this to be the case supported by the observations of intense particle fluxes 
and field-aligned currents in the region where the bright auroral intensification was observed by the UV-imager half an hour earlier.

The global field-aligned current and conductivity distributions presented in Figure 2 and the corresponding equipotential pattern presented in Figure 6 are likely to represent the prevailing electrodynamical situation during Viking orbit 360 rather accurately. The qualitative agreement between the model convection pattern and the $\mathbf{E} \times \mathbf{B}$ - drift vectors and the excellent quantitative agreement between the model and calculated potential profile along the Viking orbit is a consequence of the careful selection of realistic global input data being calibrated to the Viking and DMSP/F7 data. The asymmetry between the intense and crescent-shaped dawn convection cell (associated with the intense auroral activity and field-aligned currents) and the much weaker dusk convection cell shown in Figure 6 is consistent with earlier observations and theoretical predictions for positive IMF $B_{y}$ - conditions [Heppner, 1972; Mozer et al.,1974; Crooker, 1979].

In order to combine data from various satellites following the procedure outlined here a constructive assumption is that there is a perfect coupling between the electrodynamical parameters in the ionosphere and at satellite altitude. The good agreement between the measurements made at the two satellite altitudes, as well as between the measurements and the model input and output data, generally supports this assumption. As a result of using the assumption $\mathbb{E} \cdot \mathbf{B}=\mathbf{0}$ we found, however, in the regions of intense upward field-aligned currents, discrepancies between the model and calculated potentials (cf. Figures 6 and 7) indicative of upward parallel electric fields. The presence of upward accelerated ions, intense AKR-emissions ( $07.20 \mathrm{UT}$ ) well above the local electron gyrofrequency and absence of, or only weak signatures of electron acceleration in these regions, all indicate that Viking passed above or crossed the upper part of the acceleration regions. For the two regions of upward field-aligned currents indicated in Figure 7 the potential drops amounts to about $5-10 \mathrm{kV}$ which is within the typical range of acceleration energies for the auroral particles.

The technique presented here is in principle applicable also to more disturbed and thus time-dependent events. Several UV-images are then needed to follow 
the temporal evolution of the aurora as the satellite crosses over it. In this kind of time-dependent modelling calibration to simultaneous measurements by several spacecraft is particularly useful.

\section{Summary}

Simultaneous observations by the Viking and DMSP/F7 satellites have been used in a new method to obtain snapshot pictures of the high-latitude electrodynamics associated with a specific auroral situation viewed by the UV-imager experiment on Viking during orbit 360 . The UV-image was used to locate the regions of active intense auroras, which were represented by enhanced conductivities and upward field-aligned currents in the model input data. The global input distributions were calibrated so as to be quantitatively consistent with the conductivity and field-aligned current profiles calculated from the particle and magnetic field data along the two satellite orbits. These distributions were used together with Ohm's law to solve for the ionospheric potential distribution which was finally tested against the electric field observed by Viking. The results can be summarized as follows:

1) The model input data $\left(\Sigma\right.$ and $\left.j_{\|}\right)$are qualitatively consistent with the auroral imager data and quantitatively consistent with the Viking and DMSP/F7 observations. An important feature in the model is a current $\left(j_{\|}\right)$- conductivity coupling term.

2) There is a good agreement both qualitatively and quantitatively between the output potential distribution and the observed $\mathbf{E} \times \mathbf{B}$-drift as well as between the two corresponding potential profiles along the Viking orbit. The asymmetry between the dusk and dawn convection cells is consistent with the prevailing positive IMF $B_{y}$.

3) Potential differences between the Viking altitude and the ionosphere indicative of upward parallel electric fields are found for two time periods corresponding to Viking crossings above acceleration regions further characterized by upgoing ions, intense upward field-aligned currents and intense AKR emissions.

4) A projection of the deduced potential pattern to the equatorial plane shows for 
the last closed equipotential contour a good agreement with the average location of the plasmapause.

Acknowledgements. The authors are grateful to C.-G. Fälthammar, L. Block and P.-A. Lindqvist, The Royal Institute of Technology; R. Erlandson, The Johns Hopkins University; R. Elphinstone, University of Calgary; E. Friis-Christensen, Danish Meteorological Institute; R. Heelis, The University of Texas at Dallas and P. Rothwell, Air Force Geophysics Laboratory, for valuable comments and helpful discussions. This research was supported by the Swedish Board for Space Activities. The Viking Project is managed and operated by the Swedish Space Corporation under contract from the Swedish Board for Space Activities. The Viking magnetic field experiment was supported by the Office of Naval Research. The assistance of NASA in providing the fluxgate magnetometer sensor for the DMSP/F7 magnetic field experiment is gratefully acknowledged. We are grateful to the JHU/APL Space Department for constructing and testing the DMSP/F7 and Viking magnetic field experiments.

\section{References}

Atkinson, G. and Hutchison, D., Effect of the Day Night Ionospheric Conductivity Gradient on Polar Cap Convective Flow, J. Geophys. Res., 83, 725-729, 1978.

Block, L.P., Fälthammar, C.-G., Lindqvist, P.-A., Marklund, G., Mozer, F.S., Pedersen, A., Potemra, T.A., and Zanetti, L.J., Electric Field Measurements on Viking: First Results, Geophys. Res. Lett., 14, 435-438, 1987.

Blomberg, L.G. and Marklund, G.T., The Influence of Conductivities Consistent with Field-aligned Currents on High-latitude Convection Patterns, The Royal Institute of Technology Report TRITA-EPP-88-02, 1988a.

Blomberg, L.G. and Marklund G.T., A Numerical Model of Ionospheric Convection Derived from Field-aligned Currents and the Corresponding Conductivity, The Royal Institute of Technology Report TRITA-EPP-88-03, 1988b.

Carpenter, D.L., Whistler Studies of the Plasmapause in the Magnetosphere, 1, Temporal Variations in the Position of the Knee and Some Evidence on Plasma Motions Near the Knee, J. Geophys. Res., 71, 693-710, 1966. 
Crooker, N.U., Dayside Merging and Cusp Geometry, J. Geophys. Res., 84, 951959, 1979.

Foster, J.C., Holt, J.M., Musgrove, R.G., and Evans, D.S., Ionospheric Convection Associated with Discrete Levels of Particle Precipitation, Geophys. Res. Lett., $13,656-659,1986$.

Harel, M., Wolf, R.A., Reiff, P.H., Spiro, R.W., Burke, W.J., Rich, F.J., and Smiddy, M., Quantitative Simulation of a Magnetospheric Substorm 1, Model Logic and Overview, J. Geophys. Res., 86, 2217-2241, 1981.

Heppner, J.P., in Critical Problems of Magnetospheric Physics, Ed. by E.R. Dyer, pp. 107-122, Natl. Academy of Sci., 1972.

Iijima, T. and Potemra, T.A., The Amplitude Distribution of Field-Aligned Currents at Northern High Latitudes Observed by Triad, J. Geophys. Res., 81, 2165-2174, 1976a.

Iijima, T. and Potemra, T.A., Field-Aligned Currents in the Dayside Cusp Observed by Triad, J. Geophys. Res., 81, 5971-5979, $1976 \mathrm{~b}$.

Iijima, T. and Potemra, T.A., Large-Scale Characteristics of Field-Aligned Currents Associated with Substorms, J. Geophys. Res., 83, 599-615, 1978.

Kamide, Y., Craven, J.D., Frank, L.A., Ahn, B.-H., and Akasofu, S.-I., Modelling Substorm Current Systems Using Conductivity Distributions Inferred from DE Auroral Images, J. Geophys. Res., 91, 11235-11256, 1986.

Marklund, G.T., Raadu, M.A. and Lindqvist, P.-A., Effects of Birkeland Current Limitation on High-Latitude Convection Patterns, J. Geophys. Res., 90, 10864$10874,1985$.

Marklund, G.T., Blomberg, L.G., Potemra, T.A., Murphree, J.S., Rich, F.J., and Stasiewicz, K., A New Method to Derive "Instantaneous" High-Latitude Potential Distributions from Satellite Measurements Including Auroral Imager Data, Geophys. Res. Lett., 14, 439-442, 1987.

Mishin, V.M., Lunyushkin, S.B., Shirapov, D.Sh., and Baumjohann, W., A New Method for Generating Instantaneous Ionospheric Conductivity Models Using Ground-Based Magnetic Data, Planet. Space Sci., 34, 713-722, 1986.

Mozer, F.S., Gonzalez, W.D., Bogatt, F., Kelley, M.C., and Schutz, S.J., High- 
Latitude Electric Fields and the Three-Dimensional Interaction Between the Interplanetary and Terrestrial Magnetic Fields, J. Geophys. Res., 79, 56-63, 1974.

Sugiura, M., A Fundamental Magnetosphere-Ionosphere Coupling Mode Involving Field-Aligned Currents as Deduced from DE-2 Observations, Geophys. Res. Lett., 11, 877-880, 1984.

Wallis, D.D. and Budzinski, E.E., Empirical Models of Height-Integrated Conductivities, J. Geophys. Res., 86, 125-137, 1981. 


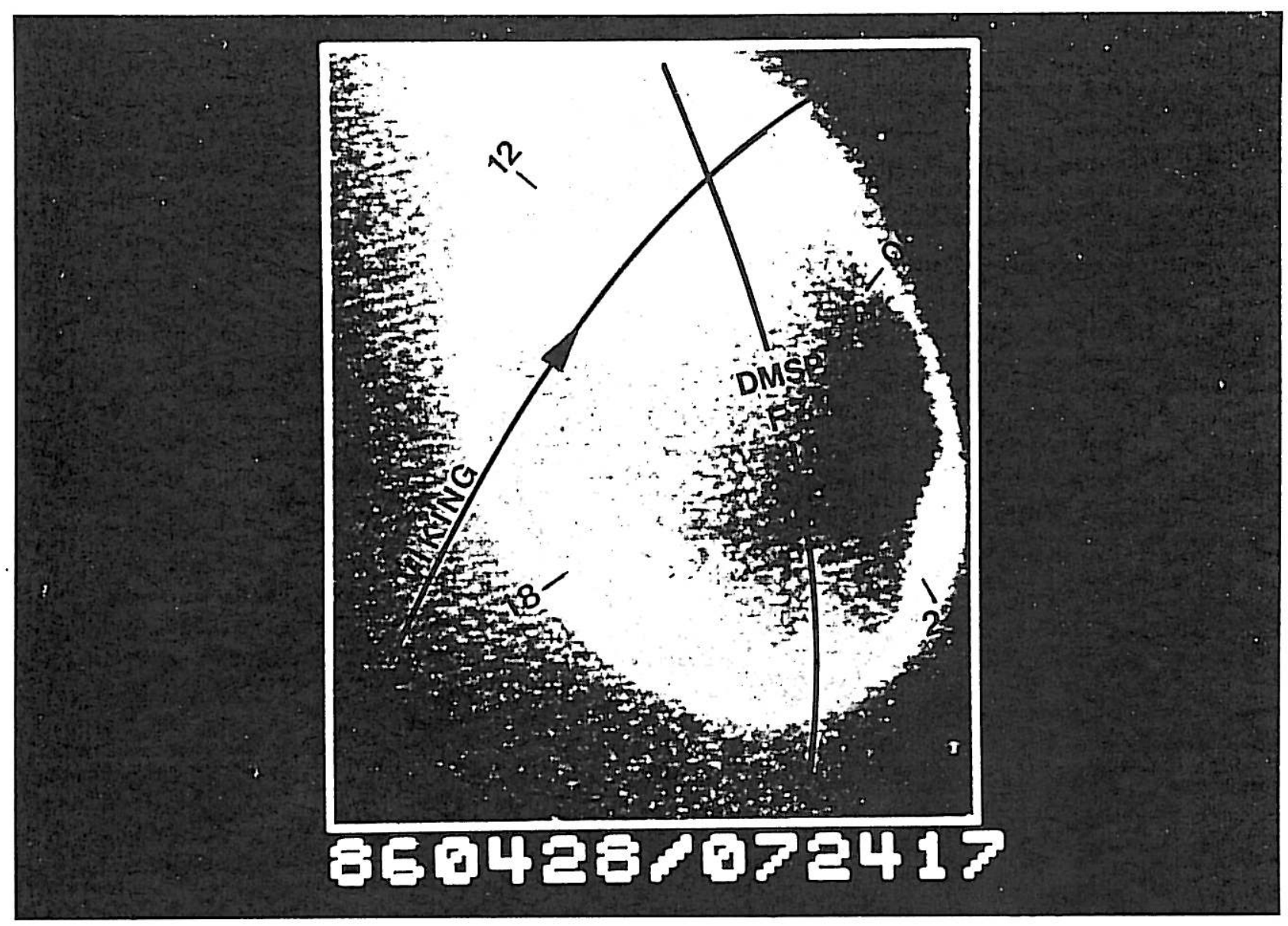

Fig. 1 The northern hemisphere auroral oval as viewed by the UV-imager experiment on Viking at 07.24 UT, orbit 360, April 28, 1986. 

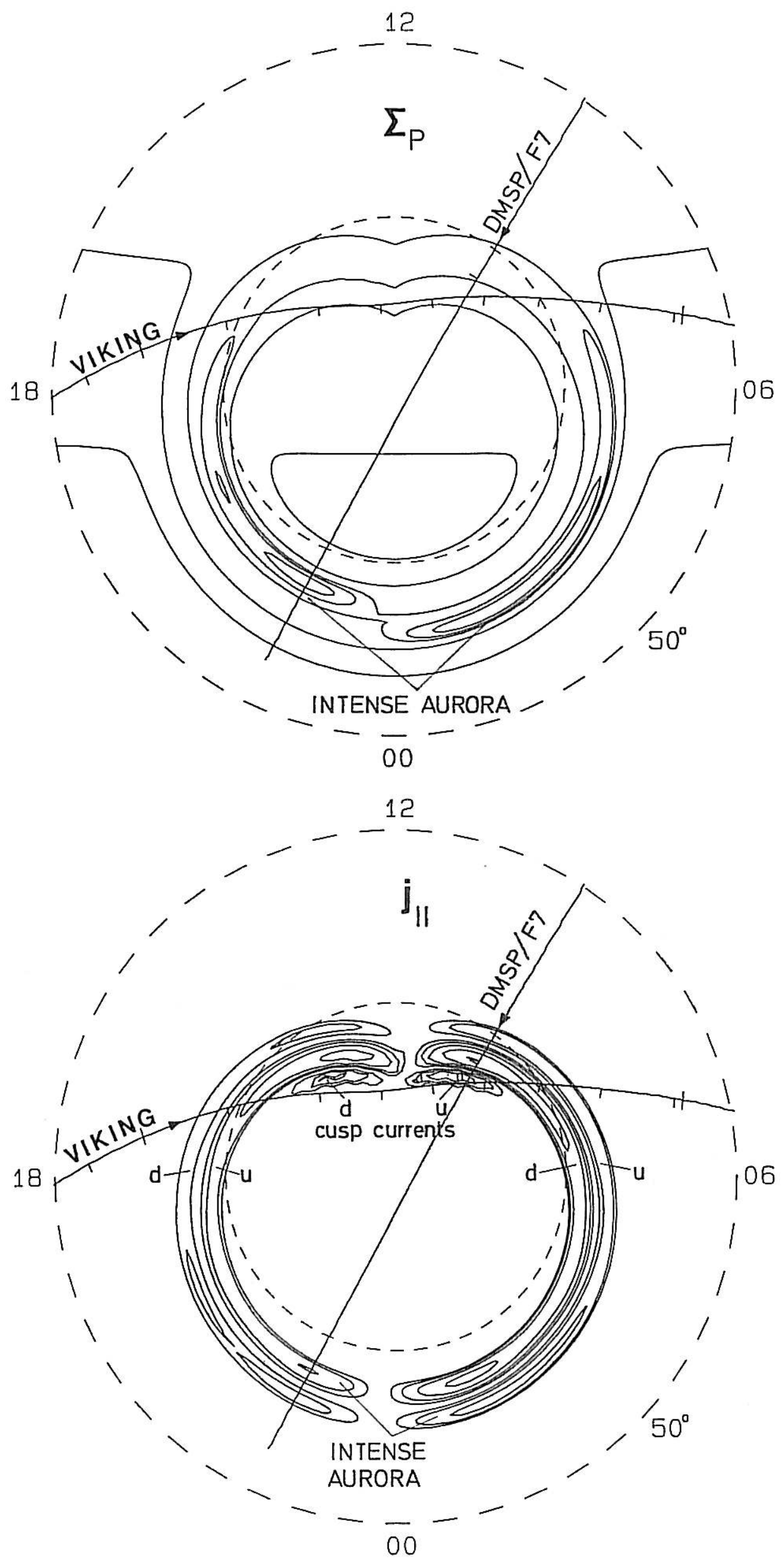

Fig. 2 Input Pedersen conductivity $\left(\Sigma_{P}\right.$, top) and input field-aligned current $\left(j_{\|}\right.$, bottom). Included are also the magnetic footpoints of the Viking (dusk-dawn) and DMSP/F7 (noon-midnight) satellites. 


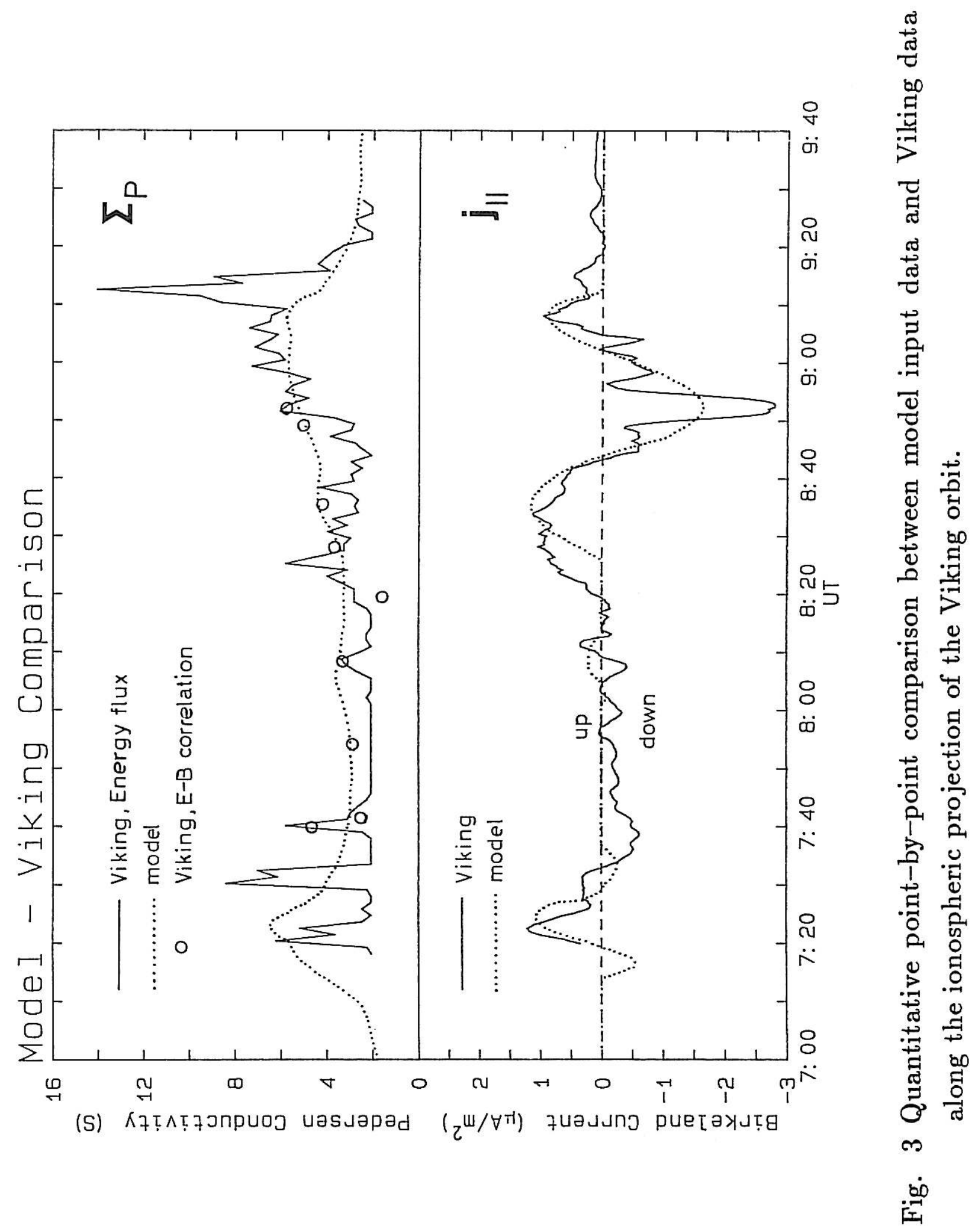



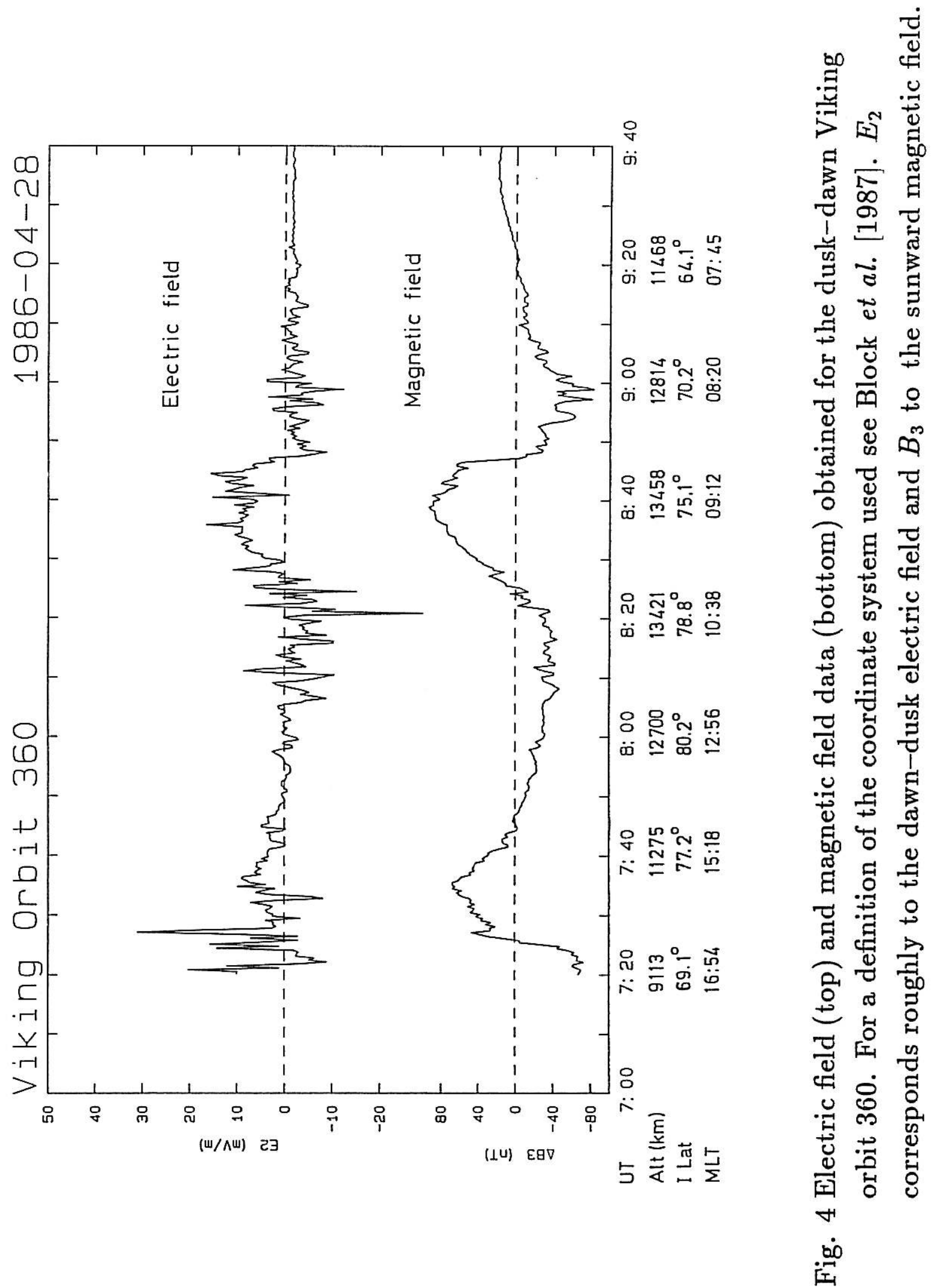


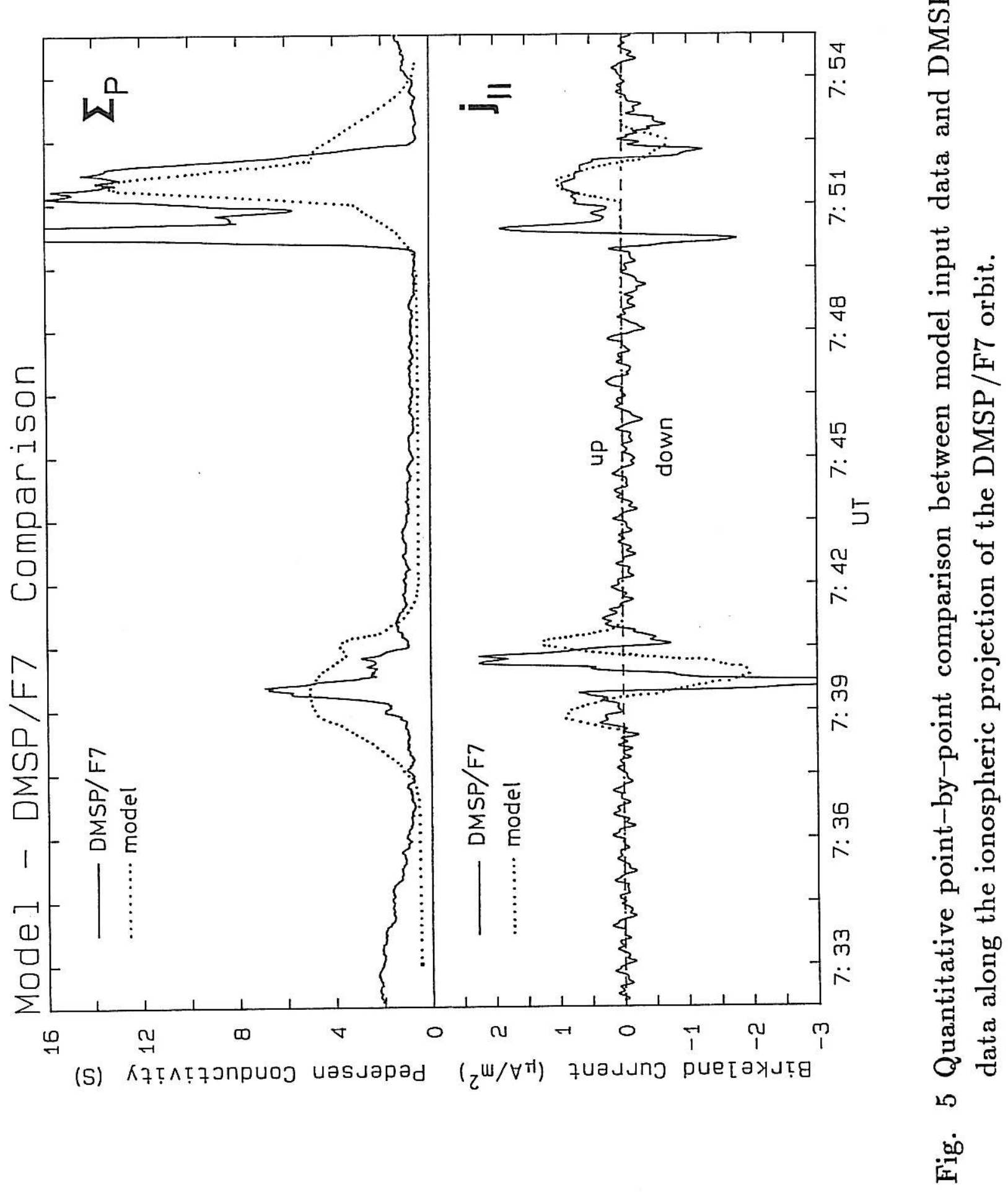




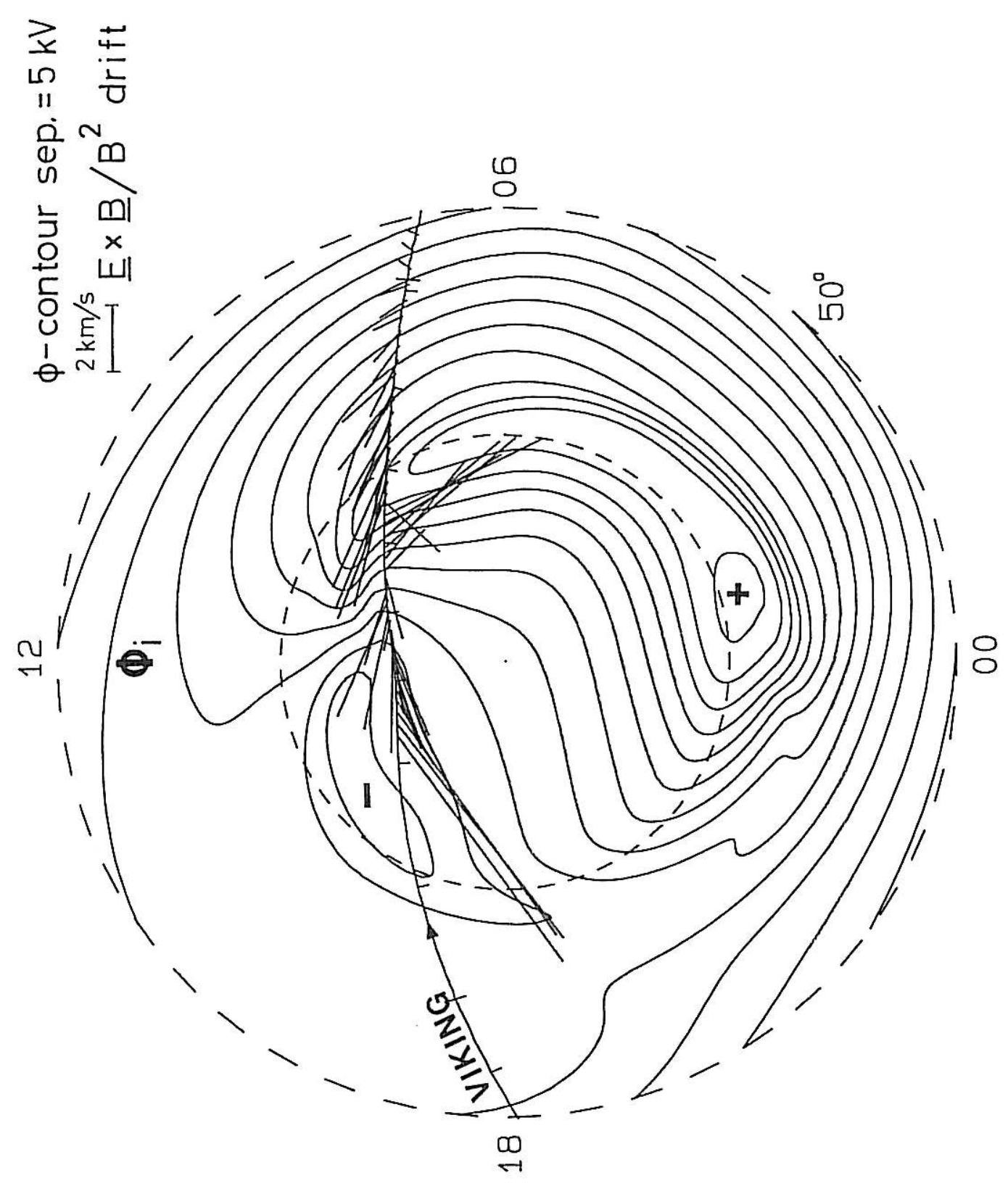

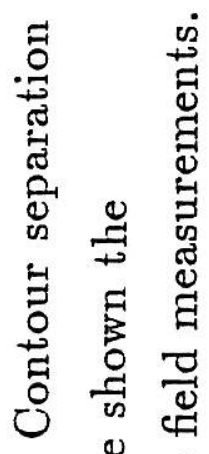

岁 㡙

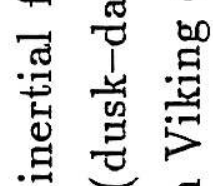

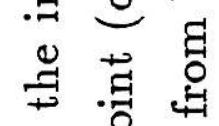

.

뎡

寻 照

紫罗。

च

严

苟

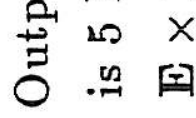

$\infty$

$\underset{10}{\infty}$ 


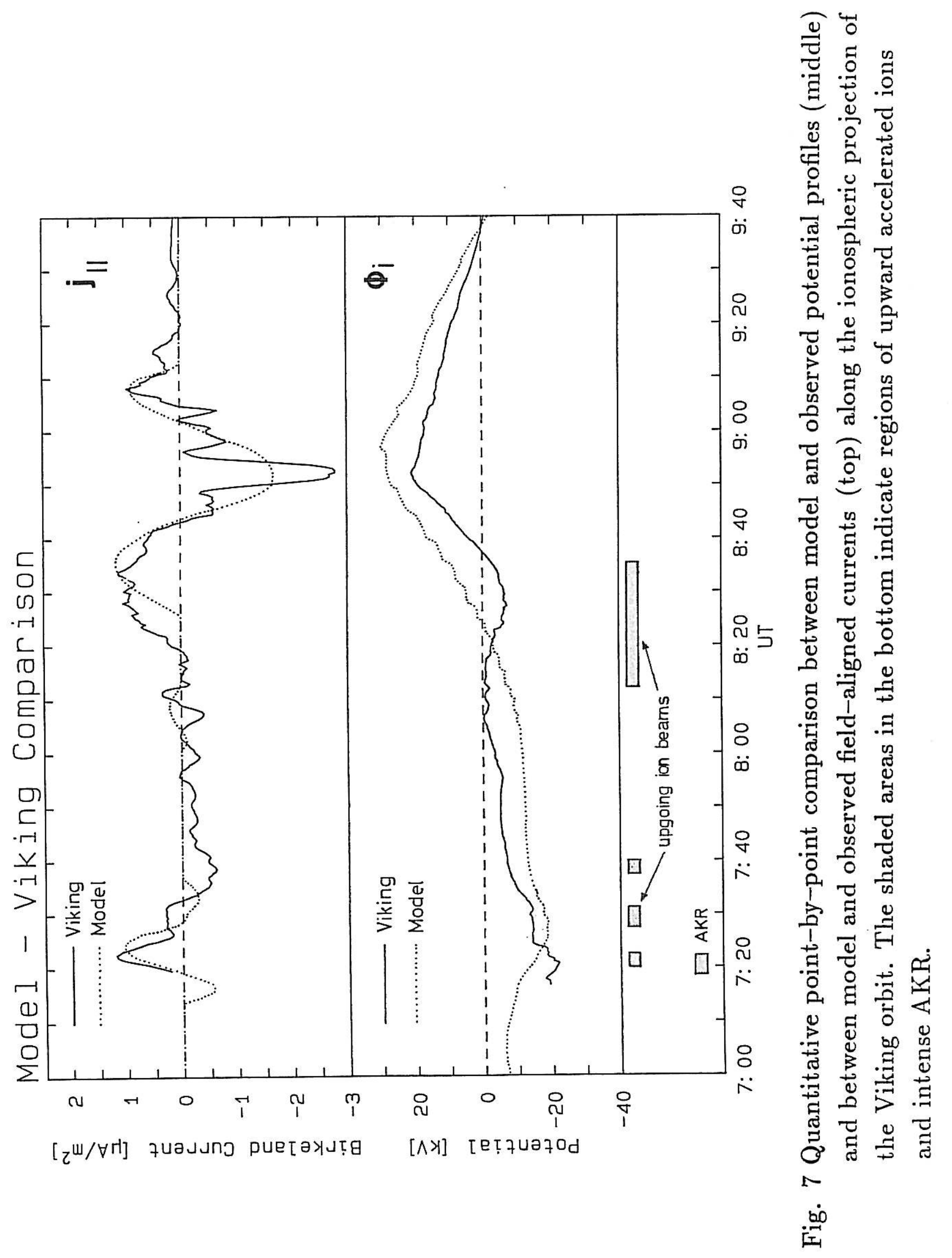




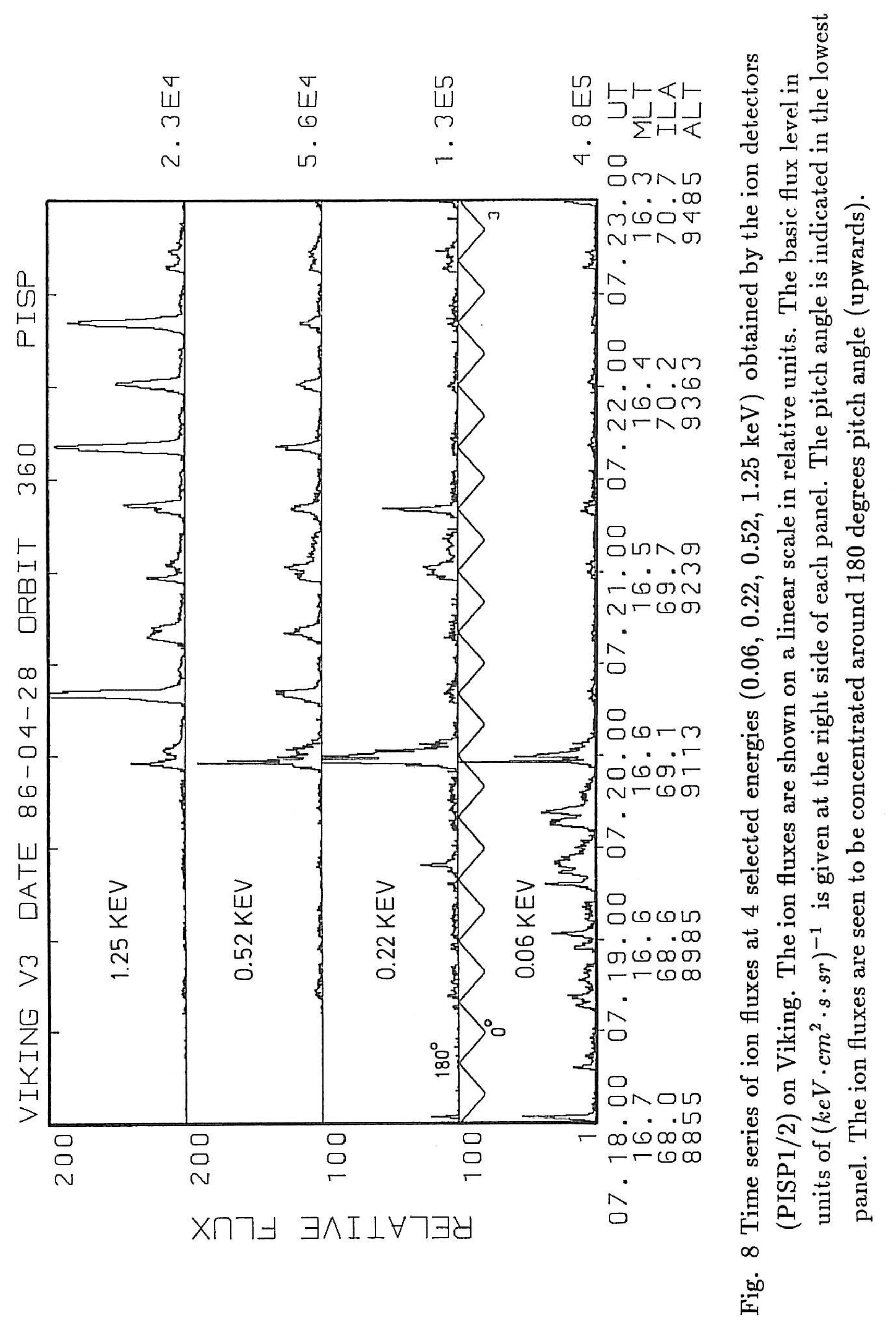




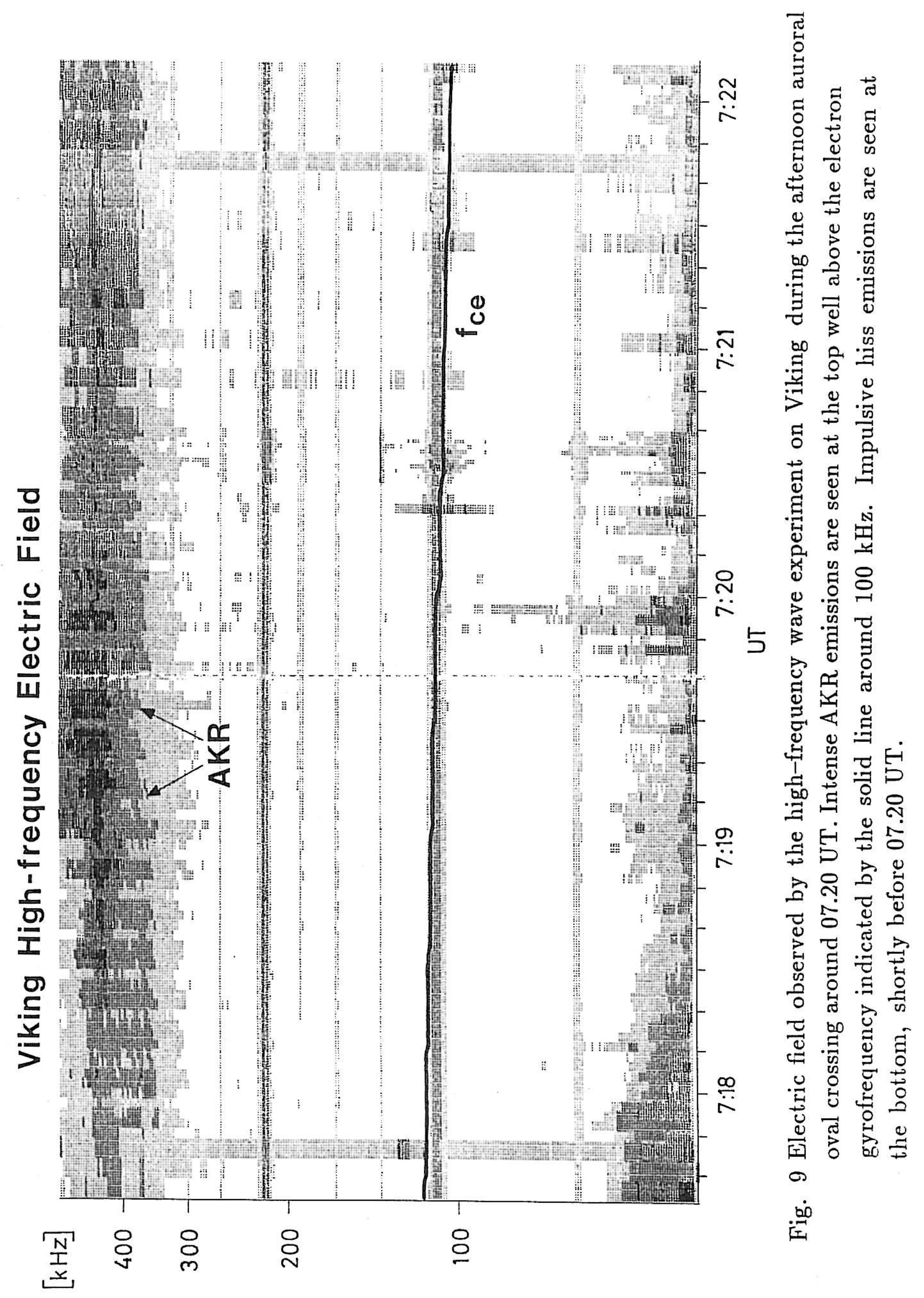




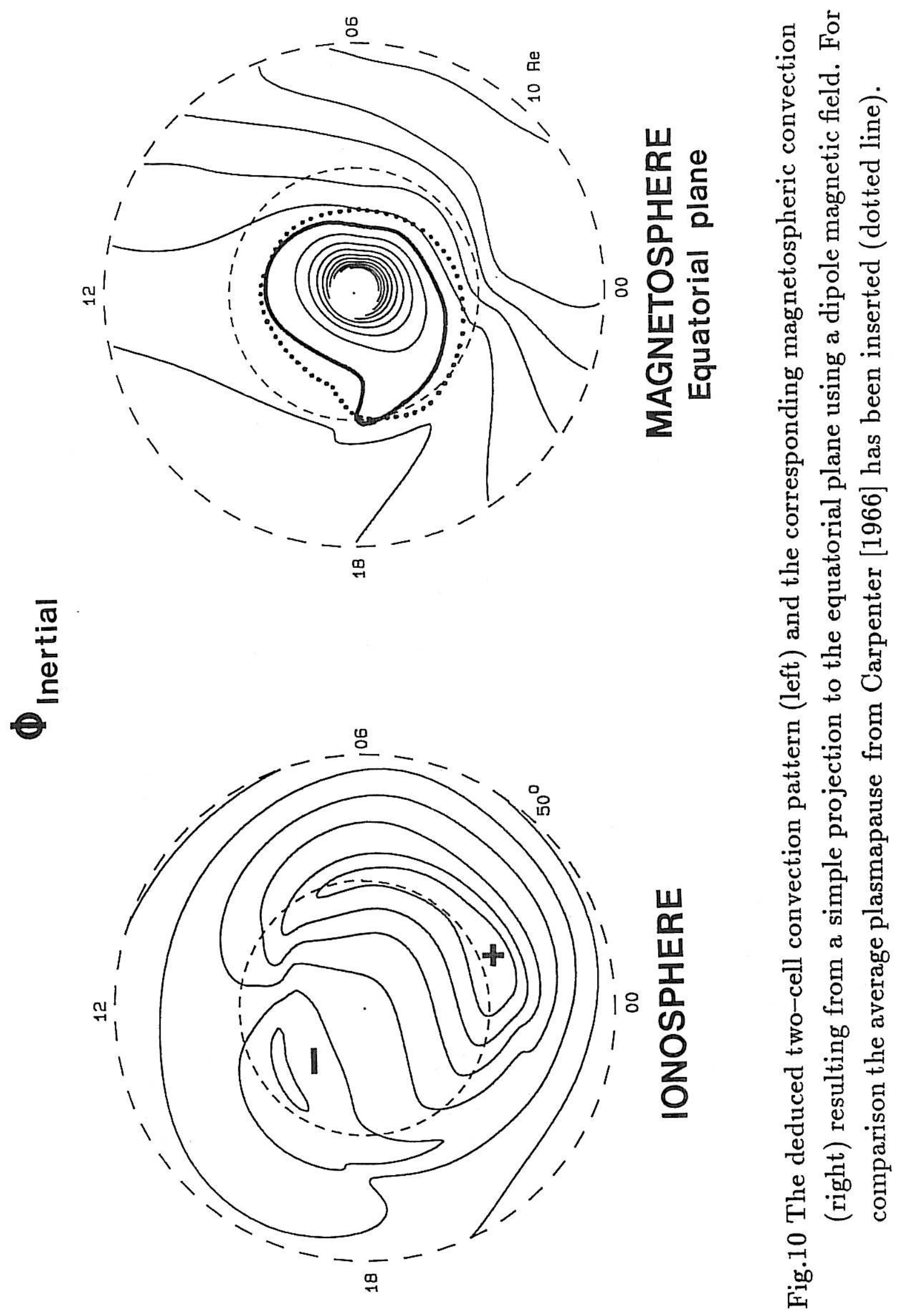


The Royal Institute of Technology, Department of Plasma Physics

S-100 44 Stockholm, Sweden

\section{SNAPSHOTS OF HIGH-LATITUDE ELECTRODYNAMICS USING VIKING AND DMSP/F7 OBSERVATIONS}

G.T. Marklund, L.G. Blomberg, K. Stasiewicz, J.S. Murphree, R. Pottelette, L.J. Zanetti, T.A. Potemra, D.A. Hardy and F.J. Rich

February 1988, 25 pp. incl. illus., in English

Simultaneous observations by the Viking and the DMSP/F7 satellites have been used in a new method to obtain snapshot pictures of the auroral electrodynamics. In particular, an "instantaneous" global equipotential (or convection) pattern is calculated using field-aligned current and conductivity distributions that are qualitatively consistent with the Viking auroral imager data and quantitatively consistent with magnetic field and particle data from the two satellites. This convection pattern which is of the normal two-cell type, with a weak dusk cell and a strong, elongated crescent-shaped dawn cell (consistent with positive IMF $B_{y}$ ) agrees well with the Viking electric field data. The model and the observed potential profiles agree nicely along the entire Viking orbit except for two intervals above acceleration regions where deviations are to be expected (due to parallel electric fields). These regions are characterized by: U-shaped potential minima, upward field-aligned currents, upgoing ion beams and relatively intense AKR. The model results are thus consistent with the Viking observations not only on a global scale but also on the scale size of the auroral acceleration regions. The corresponding convection in the magnetosphere is illustrated by a simple projection of the deduced two-cell convection pattern to the equatorial plane. From this the instantaneous location of the plasmapause is inferred.

Key words: High-latitude electrodynamics, Viking observations, Auroral imaging, Auroral acceleration, Ionospheric convection, Ionospheric modelling 